\title{
Aplicación de una aproximación metodológica simple para el análisis de las desigualdades: El caso de la mortalidad infantil en Chile
}

\author{
PATRICIA FRENZ1, CLAUDIA GONZÁLEZ ${ }^{2 a}$
}

\section{Application of a simple methodological approach to analyze health inequalities: The case of infant mortality in Chile}

Background: The infant mortality gradient by maternal education is a good indicator of the health impact of the social inequalities that prevail in Chile. Aim: To propose a systematic method of analysis, using simple epidemiological measures, for the comparison of differential health risks between social groups that change over time. Material and Methods: Data and statistics on births and infant deaths, obtained from the Ministry of Health, were used. Five strata of maternal schooling were defined and various measures were calculated to compare infant mortality, according to maternal education in the periods 1998-2001 and 2001-2003. Results: Of particular interest is the distinction between a measure of effect, Relative Risk $(R R)$, which indicates the size of the gap between socioeconomic extremes and the etiological strength of low maternal schooling on infant mortality, and a measure of global impact, the Population Attributable Risk (PAR\%), which takes into account the whole socioeconomic distribution and permits comparisons over time independently of the variability in the proportions of the different social strata. The comparison of these measures in the two periods studied, reveals an increase in the infant mortality gap between maternal educational extremes measured by the RR, but a stabilization in the population impact of low maternal schooling. Conclusions: These results can be explained by a decline in the proportion of mothers in the lowest educational level and an increase in the proportion in the highest group.

(Rev Med Chile 2010; 138: 1157-1164).

Key words: Health risk indicators; Infant mortality; Risk assessment.

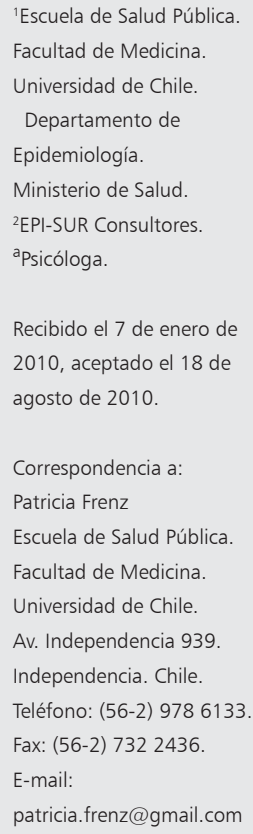<smiles>[2H]</smiles>
isminuir las desigualdades en salud" es uno de los cuatro grandes Objetivos Sanitarios para la Década 2000-2010 definidos por el Ministerio de Salud ${ }^{1}$. Este objetivo expresa el principio de equidad de la Reforma de la Salud $^{2}$, en el sentido de minimizar las diferencias evitables, innecesarias e injustas en la salud entre grupos sociales ${ }^{3}$. El primer objetivo de impacto asociado a este desafío es "Reducir la brecha de mortalidad infantil entre grupos educacionales extremos; siendo su meta reducir en un $10 \%$ el diferencial de mortalidad infantil entre hijos de madres de grupos educacionales extremos" (1:227).

La medida de desigualdad usada en esta meta fue el riesgo relativo (RR), usando la razón de tasas de los grupos educacionales extremos. Según el reporte, para el período 1998-2000 el riesgo de morir era 2,6 veces mayor para menores de un año de madres con hasta 3 años de escolaridad, en comparación con el riesgo de los niños de madres 
con 13 o más años de educación. Por lo tanto, reducir en $10 \%$ esta diferencia significaba llegar a un RR de 2,34.

La evaluación del cumplimiento de esta meta se realizó en dos momentos, junto con las otras metas de los Objetivos Sanitarios: a mitad del período $(2005)^{4}$ y al final de la década, proceso que está llevando a cabo el Ministerio de Salud en la actualidad. En 2005 la evaluación de la meta de reducción de la brecha de mortalidad infantil mostró que, si bien hay reducciones en la tasa global de mortalidad infantil, así como en las tasas específicas de cada grupo educacional, el RR entre grupos extremos aumentó a 3,0 para el período 2001-2003 (4: 44-45).

Algunos estudios han examinado las desigualdades en la mortalidad infantil en Chile por grupos de educación materna, estatus ocupacional y geográficos, usando el $\mathrm{RR}^{5,6}$, pero no se ha reportado un análisis con una medida de resumen del impacto de la gradiente social en la tasa poblacional.

El presente trabajo tiene como objetivo proponer una aproximación metodológica, que complemente el análisis de desigualdades sociales de la mortalidad infantil en Chile. Para ello, utiliza un método simple de análisis que permite profundizar en la interpretación de las tendencias del riesgo de morir de los menores del año, por estratos de escolaridad materna que varían en el tiempo. El análisis ilustra algunas consideraciones metodológicas y conceptuales importantes para el estudio de desigualdades en salud ${ }^{7}$.

\section{Material y Método}

Su utilizó un método de análisis en cinco pasos, aplicando medidas epidemiológicas simples para medir cambios en la magnitud de desigualdades sanitarias en el tiempo, que toma en cuenta orientaciones y métodos desarrollados por varios autores: Mackenbach y Kunst ${ }^{7}$, Shaw y cols $^{8}$, y Scheider y cols 9 . La descripción de las medidas epidemiológicas usadas y su significado se resumen en la Tabla 1.

El análisis usó los datos y estadísticas de mortalidad infantil relacionados con el objetivo de reducir la brecha de mortalidad infantil en $10 \%$ entre hijos de madres de escolaridad extrema, reportados en la evaluación de los Objetivos Sanitarios de $2005^{4}$. En dicha evaluación se definieron cinco estratos de escolaridad materna como medi- da de posición socioeconómica de los niños: 0-3 años, 4-6 años, 7-9 años, 10-12 años y 13+ años de escolaridad (4:43).

A partir de esa información, se siguieron los siguientes pasos:

1. Se calculó y graficó la proporción de nacimientos y muertes infantiles, según grupo educacional de la madre para los períodos 1998-2000 y 2001-2003, en base al número de muertes y nacimientos por grupo de escolaridad materna, reportado en las tablas 13 y 14 de la evaluación de los Objetivos Sanitarios (4:43-44). Este simple cálculo permite analizar el tamaño de los grupos sociales, su cambio en los dos períodos y observar si la proporción de muertes de cada estrato es mayor o menor que la representación del grupo en los nacimientos.

2. Se calcularon y graficaron las tasas de mortalidad infantil total y específicas para cada grupo social y su variación entre los dos períodos, lo que permite observar la gradiente social y su evolución.

A las tasas específicas por grupo de escolaridad y los Riesgos Relativos informados por el Ministerio de Salud, se agregaron los siguientes cálculos: a) Porcentaje de reducción de las tasas específicas por grupo de escolaridad materna entre los dos períodos; b) Riesgo Absoluto o atribuible (RA), que es la diferencia entre las tasas de mortalidad infantil. En los cálculos se tomó como grupo de referencia el estrato de 13 o más años.

3. Se analizaron las brechas absolutas (RA) y relativas (RR) de los grupos educacionales en relación al grupo de referencia, lo que permite medir la magnitud del efecto de la desventaja social y su evolución en términos absolutos y relativos.

4. Se redefinieron los grupos educacionales, para acercarse a una distribución por quintiles y calcular las tasas en este nuevo escenario, lo que permite observar la importancia que tiene la definición de los estratos de jerarquía social en la magnitud de la brecha.

5. Finalmente, se calculó una medida de resumen del impacto de la gradiente social en salud en la población: el Riesgo Atribuible Poblacional Porcentual (RAP\%), que permite medir el impacto total de la desventaja social sobre la mortalidad infantil en Chile, tomando en cuenta el tamaño de los diferentes grupos sociales ${ }^{7}$. 
Tabla 1. Medidas de desigualdad social en salud de cálculo simple

\begin{tabular}{|c|c|c|c|c|}
\hline Medida & Fórmula de cálculo & Interpretación & Fortalezas & Limitaciones \\
\hline $\begin{array}{l}\text { Distribución } \\
\text { \% de casos } \\
\text { y población } \\
\text { por grupo } \\
\text { social }\end{array}$ & $\begin{array}{l}\text { Distribución \% del gru- } \\
\text { pos sociales en la po- } \\
\text { blación y la distribución } \\
\text { de los casos por grupo } \\
\text { social } \\
\text { Ej. \% nacimientos y de } \\
\text { muertes infantiles, por } \\
\text { grupos de escolaridad }\end{array}$ & $\begin{array}{l}\text { Si no hay diferencias de } \\
\text { riesgo de enfermedad o } \\
\text { muerte entre los grupos } \\
\text { sociales, la proporción } \\
\text { de eventos según grupo } \\
\text { debe ser igual a la pro- } \\
\text { porción del grupo en la } \\
\text { población. }\end{array}$ & $\begin{array}{l}\text { Presentación simple de } \\
\text { desproporciones que re- } \\
\text { flejan cargas desiguales } \\
\text { de problemas de salud } \\
\text { en los diferentes grupos } \\
\text { sociales. Toma en cuenta } \\
\text { en tamaño de los gru- } \\
\text { pos en la población. }\end{array}$ & $\begin{array}{l}\text { No resume en una ci- } \\
\text { fra la magnitud de la } \\
\text { desproporcionalidad. } \\
\text { Las medidas de despro- } \\
\text { porcionalidad como el } \\
\text { Índice de Concentración } \\
\text { entregan una medida } \\
\text { de resumen, pero son } \\
\text { más difíciles de calcular } \\
\text { e interpretar. }\end{array}$ \\
\hline $\begin{array}{l}\text { Tasas especí- } \\
\text { ficas por } \\
\text { grupo social }\end{array}$ & $\begin{array}{l}\text { Casos de cada grupo } \\
\text { social por población en } \\
\text { riesgo de cada grupo so- } \\
\text { cial (x un amplificador). } \\
\text { Ej. muertes infantiles } \\
\text { según grupo de escolari- } \\
\text { dad materna por mil na- } \\
\text { cidos vivos según grupo } \\
\text { de escolaridad materna }\end{array}$ & $\begin{array}{l}\text { La tasa específica de } \\
\text { cada grupo social repre- } \\
\text { senta la probabilidad del } \\
\text { evento (el riesgo) de ese } \\
\text { grupo social. }\end{array}$ & $\begin{array}{l}\text { Las tasas específicas, } \\
\text { especialmente su pre- } \\
\text { sentación gráfica, per- } \\
\text { miten observar posibles } \\
\text { gradientes sociales en } \\
\text { salud. }\end{array}$ & $\begin{array}{l}\text { Por si sólo no son medi- } \\
\text { das precisas de la mag- } \\
\text { nitud del efecto de la } \\
\text { desventaja social en el } \\
\text { riesgo de morir. }\end{array}$ \\
\hline $\begin{array}{l}\text { Riesgo } \\
\text { absoluto o } \\
\text { atribuible }\end{array}$ & $\begin{array}{l}\text { Tasa del grupo social } \\
\text { bajo menos la Tasa del } \\
\text { grupo social alta }\end{array}$ & $\begin{array}{l}\text { Estimación del efecto } \\
\text { absoluto atribuible a la } \\
\text { exposición a la desven- } \\
\text { taja social. }\end{array}$ & $\begin{array}{l}\text { Es un indicador de la } \\
\text { magnitud del problema, } \\
\text { que refleja su importan- } \\
\text { cia para la salud pública. }\end{array}$ & $\begin{array}{l}\text { Debe complementarse } \\
\text { con la medición del } \\
\text { efecto relativo. Al com- } \\
\text { parar los grupos sociales } \\
\text { extremos no toma en } \\
\text { cuenta los otros grupos } \\
\text { (la gradiente social). No } \\
\text { entrega información so- } \\
\text { bre el tamaño del grupo } \\
\text { social }\end{array}$ \\
\hline $\begin{array}{l}\text { Riesgo } \\
\text { relativo }\end{array}$ & $\begin{array}{l}\text { Tasa del grupo social } \\
\text { bajo divido por Tasa del } \\
\text { grupo social alto }\end{array}$ & $\begin{array}{l}\text { Estimación del efecto } \\
\text { que tiene la desventaja } \\
\text { social sobre el riesgo } \\
\text { de enfermar o morir. } \\
\text { Corresponde a la brecha } \\
\text { por extremos sociales. }\end{array}$ & $\begin{array}{l}\text { Es considerado un me- } \\
\text { jor indicador del efecto } \\
\text { causal que la diferencia } \\
\text { absoluta. }\end{array}$ & $\begin{array}{l}\text { Debe complementarse } \\
\text { con la diferencia absolu- } \\
\text { ta de tasas. Al comparar } \\
\text { los extremos sociales no } \\
\text { considera la gradien- } \\
\text { te social. No toma en } \\
\text { cuenta el tamaño de los } \\
\text { grupos. }\end{array}$ \\
\hline $\begin{array}{l}\text { Riesgo } \\
\text { atribuible } \\
\text { poblacional } \\
\%\end{array}$ & $\begin{array}{l}\text { ((Tasa promedio pobla- } \\
\text { cional menos Tasa del } \\
\text { grupo social alto)/Tasa } \\
\text { promedio poblacional } \\
\text { x } 100\end{array}$ & $\begin{array}{l}\text { La proporción de muer- } \\
\text { tes o enfermedades que } \\
\text { se atribuye a la desven- } \\
\text { taja social relativa de } \\
\text { todos grupos (en rela- } \\
\text { ción al grupo más alto). } \\
\text { También interpretado } \\
\text { como el porcentaje de } \\
\text { muertes o enfermeda- } \\
\text { des que se evitaría si se } \\
\text { podría eliminar el efecto } \\
\text { de la desventaja social } \\
\text { en todos los grupos de } \\
\text { la población. }\end{array}$ & $\begin{array}{l}\text { Es una medida de resu- } \\
\text { men del impacto total } \\
\text { de la desventaja social } \\
\text { en la población, que } \\
\text { toma en cuenta la gra- } \\
\text { diente social y el tamaño } \\
\text { de los grupos sociales. } \\
\text { Es un buen indicador } \\
\text { de la importancia para } \\
\text { la salud pública de una } \\
\text { exposición. }\end{array}$ & $\begin{array}{l}\text { A pesar de ser fácil de } \\
\text { calcular y sus ventajas } \\
\text { conceptuales, su uso no } \\
\text { ha sido tan difundido y } \\
\text { puede ser difícil de ex- } \\
\text { plicar a un tomador de } \\
\text { decisiones. }\end{array}$ \\
\hline
\end{tabular}

Fuente: Elaboración propia a partir de Mackenbach y Kunst ${ }^{7}$, Shaw y col${ }^{8}$, y Schneider y col ${ }^{9}$. 
Esta medida es una estimación de la fracción etiológica del riesgo de morir en una población atribuible a una exposición, que en este caso es la desventaja social relativa. Otra manera de interpretarla es que corresponde a la proporción de muertes infantiles que se podrían evitar, si fuera posible eliminar las múltiples exposiciones y vulnerabilidades asociadas a pertenecer a los grupos de escolaridad menores de 13 años.

RAP\% $=$ (tasa media poblacional - tasa de referencia $)$ /tasa poblacional ${ }^{*} 100$

\section{Resultados}

Paso 1. Análisis de proporciones de nacimientos $y$ muertes infantiles por grupo educacional de la madre

Al comparar el porcentaje de nacimientos entre los grupos educacionales (Tabla 2), se observa que en ambos períodos, el grupo de 13 y más años concentra alrededor del 20\% de los nacimientos, mientras que el de 0-3 años representa menos del
2\%. Asimismo, entre los dos períodos estudiados, la proporción de nacimientos del grupo de escolaridad baja disminuye, mientras que la del grupo de escolaridad alta, crece. Disminuyen también las proporciones de nacimientos de los grupos de 4-6 y 7-9 años de escolaridad. En relación a las muertes infantiles, en ambos períodos la proporción de muertes ocurridas en el grupo 0-3 años de escolaridad materna es alrededor de $3 \%$ y en el grupo de escolaridad más alta es $13 \%$.

Al comparar las proporciones de muertes y nacimientos infantiles de los grupos, se evidencia una situación de desproporcionalidad. Así, la proporción de muertes en el grupo de menor escolaridad es superior a la proporción de nacimientos; mientras que la proporción de muertes del grupo de 13 y más años de escolaridad materna es menor que la proporción de nacimientos de este grupo. Esta situación muestra el mayor riesgo de morir del grupo de escolaridad baja; lo mismo se observa para los dos grupos que le siguen hasta los 9 años de escolaridad materna. La Figura 1 ilustra la desproporcionalidad en los dos períodos.

Tabla 2. Distribución porcentual de defunciones, nacidos vivos y tasas de mortalidad infantil, según escolaridad de la madre (1998-2000 y 2001-2003)

\begin{tabular}{|c|c|c|c|c|c|c|}
\hline \multirow{2}{*}{$\begin{array}{l}\text { Grupo educacional } \\
\text { de la madre }\end{array}$} & \multicolumn{3}{|c|}{ Muertes infantiles } & \multicolumn{3}{|c|}{ Nacimientos } \\
\hline & $\mathbf{n}$ & $\%$ & $\%$ acumulado & $\mathbf{n}$ & $\%$ total & $\%$ acumulado \\
\hline \multicolumn{7}{|c|}{ Muertes infantiles y Nacimientos en Chile 1998-2000 } \\
\hline 0 a 3 años & 227 & 3,05 & 3,05 & 14.543 & 1,93 & 1,93 \\
\hline 4 a 6 años & 984 & 13,21 & 16,26 & 63.931 & 8,47 & 10,39 \\
\hline 7 a 9 años & 1.507 & 20,23 & 36,48 & 123.788 & 16,40 & 26,79 \\
\hline 10 a 12 años & 3.809 & 51,13 & 87,61 & 399.066 & 52,86 & 79,65 \\
\hline $13 y+$ años & 923 & 12,39 & 100,00 & 153.619 & 20,35 & 100,00 \\
\hline Total & 7.450 & 100,00 & 100,00 & 754.947 & 100,00 & 100,00 \\
\hline \multicolumn{7}{|c|}{ Muertes infantiles y Nacimientos en Chile 2001-2003 } \\
\hline 0 a 3 años & 161 & 2,72 & 2,72 & 10.666 & 1,48 & 1,48 \\
\hline 4 a 6 años & 657 & 11,10 & 13,82 & 49.287 & 6,85 & 8,34 \\
\hline 7 a 9 años & 1.069 & 18,05 & 31,87 & 106.328 & 14,78 & 23,12 \\
\hline 10 a 12 años & 3.227 & 54,50 & 86,37 & 392.997 & 54,64 & 77,76 \\
\hline $13 y+$ años & 807 & 13,63 & 100,00 & 159.963 & 22,24 & 100,00 \\
\hline Total & 5.921 & 100,00 & 100,00 & 719.241 & 100,00 & 100,00 \\
\hline
\end{tabular}

Fuente: Elaboración propia a partir de los datos del MINSAL 2006. 


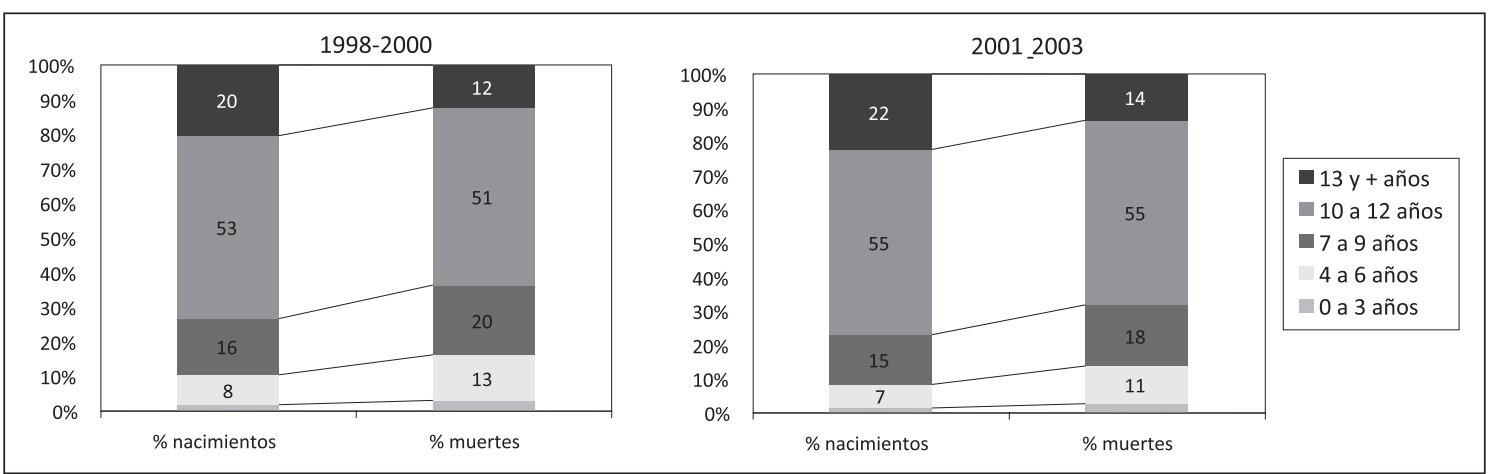

Figura 1. Proporción de nacidos vivos y muertes infantiles según escolaridad materna, Chile 1998-2000 y 2001-2003.

Pasos 2 y 3. Tasas de mortalidad infantil según grupo de escolaridad y riesgos absolutos y relativos

Las tasas de mortalidad infantil por grupo de escolaridad muestra la existencia de una gradiente social: a menor educación de la madre, mayor es la tasa de mortalidad infantil específica (Tabla 3 y Figura 2).

La revisión de los cambios porcentuales en las tasas de mortalidad infantil, según escolaridad de la madre en los dos períodos estudiados, muestra que todos los grupos disminuyeron su mortalidad; sin embargo, el grupo que muestra la menor disminución es el de menor escolaridad. Al analizar la variación porcentual según los componentes de la mortalidad infantil, se observa que el grupo de escolaridad materna más baja muestra un leve aumento en la mortalidad neonatal precoz y post-neonatal. Sin embargo, resulta llamativa la disminución que presenta este grupo en la mortalidad neonatal tardía (46\%), siendo éste el grupo de escolaridad con la mayor disminución en este componente.

El análisis de la variación en los riesgos absolutos y relativos entre el grupo de menor (0-3 años) y el de mayor escolaridad materna (13 y más años) entre los dos períodos estudiados, muestra que aumentaron las diferencias en la mortalidad infantil total y los componentes neonatal precoz y post-neonatal. Nuevamente, destaca la situación de la neonatal tardía que muestra un descenso en el riesgo absoluto y relativo del grupo de menor escolaridad de la madre.

\section{Paso 4. Análisis según redefinición de tramos de grupos educacionales}

Para aproximarse a una comparación por quintiles extremos de escolaridad, se reagruparon los tres primeros grupos de escolaridad de la madre en uno solo: de 0 a 9 años, ya que este tramo concentra

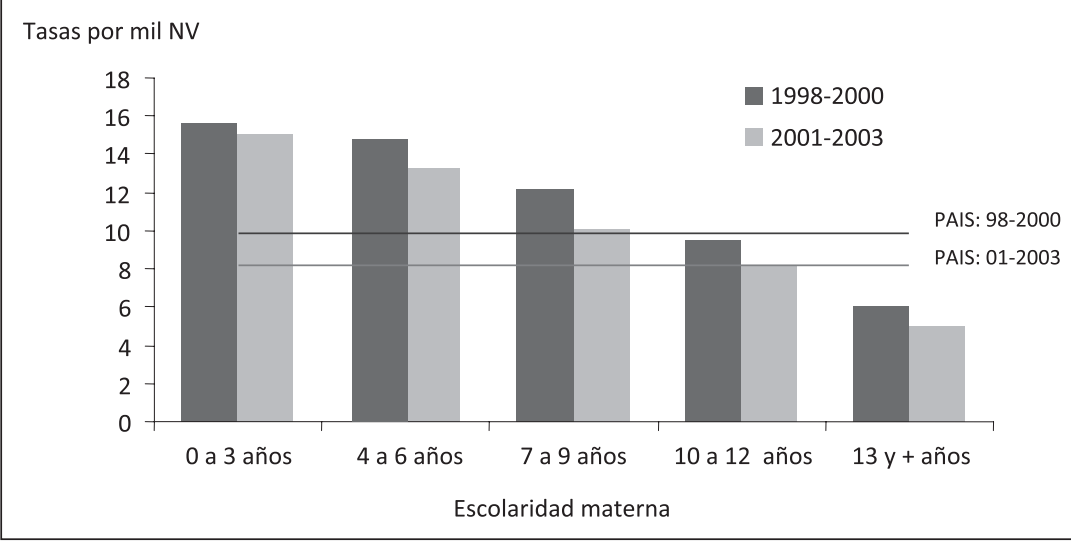

Figura 2. Gradiente de tasas de mortalidad infantil según años de escolaridad materna, Trienios $1998-2000$ y $2001-2003$. 
el 25\% de los nacimientos (Tabla 4). Los otros dos grupos se mantuvieron (10 a 12 años y 13 y más años de educación), quedando por lo tanto tres grupos de escolaridad materna.

Luego, se calcularon para los dos períodos las tasas de mortalidad infantil para el nuevo grupo de hijos de madres con 0 a 9 años de educación y se compararon con las tasas el grupo de $13+$ años, mediante los riesgos absolutos y relativos. Los resultados se presentan en la Tabla 4.

La comparación inicial, con los tramos originales, mostraba que las diferencias relativas y absolutas entre los grupos extremos (0-3 y $13+$ años) habían aumentado; pero al ampliar el gru-

Tabla 3. Tendencias de desigualdades en Mortalidad Infantil, según cambios en riesgo absoluto y riesgo relativo, comparaciones trienios 1998-2000 y 2001-2003

\begin{tabular}{|c|c|c|c|c|c|c|c|c|c|}
\hline \multirow[t]{2}{*}{$\begin{array}{l}\text { Grupos de } \\
\text { escolaridad } \\
\text { materna }\end{array}$} & \multirow{2}{*}{$\begin{array}{l}\text { Tasa } \\
\text { de MI } \\
1990- \\
2000\end{array}$} & \multirow{2}{*}{$\begin{array}{l}\text { Tasa } \\
\text { de MI } \\
2001- \\
2003\end{array}$} & \multirow[t]{2}{*}{$\begin{array}{c}\text { Cambio } \\
\%\end{array}$} & \multirow{2}{*}{$\begin{array}{c}\text { RA } \\
\text { baja/alta } \\
1998- \\
2000\end{array}$} & \multirow{2}{*}{$\begin{array}{c}\text { RA } \\
\text { baja/alta } \\
2001- \\
2003\end{array}$} & \multirow{2}{*}{$\begin{array}{c}\text { RR } \\
\text { baja/alta } \\
1998- \\
2000\end{array}$} & \multirow{2}{*}{$\begin{array}{c}\text { RR } \\
\text { baja/alta } \\
2001- \\
2003\end{array}$} & \multicolumn{2}{|c|}{$\begin{array}{c}\text { Tendencia } \\
\text { desigualdad según } \\
\text { cambio RA y RR }\end{array}$} \\
\hline & & & & & & & & $\mathbf{R A}$ & $\mathbf{R R}$ \\
\hline \multicolumn{10}{|c|}{ Mortalidad Infantil Total } \\
\hline Total & 9,9 & 8,2 & $-17,2$ & & & & & & \\
\hline 0 a 3 años & 15,6 & 15,1 & $-3,2$ & 9,60 & 10,10 & 2,60 & 3,02 & & 7 \\
\hline 4 a 6 años & 15,4 & 13,3 & $-13,6$ & 9,40 & 8,30 & 2,57 & 2,66 & & $\uparrow$ \\
\hline 7 a 9 años & 12,2 & 10,1 & $-17,2$ & 6,20 & 5,10 & 2,03 & 2,02 & $\nabla$ & - \\
\hline 10 a 12 años & 9,5 & 8,2 & $-13,7$ & 3,50 & 3,20 & 1,58 & 1,64 & & $\uparrow$ \\
\hline 13 y + años & 6,0 & 5 & $-16,7$ & 0 & 0 & 1,00 & 1,00 & & \\
\hline \multicolumn{10}{|c|}{ Neonatal Precoz } \\
\hline Total & 4,5 & 4,0 & $-11,1$ & & & & & & \\
\hline 0 a 3 años & 5,1 & 5,6 & 9,8 & 1,50 & 2,60 & 1,42 & 1,87 & & \\
\hline 4 a 6 años & 5,9 & 5,5 & $-6,8$ & 2,30 & 2,50 & 1,64 & 1,83 & & $\uparrow$ \\
\hline 7 a 9 años & 4,8 & 4,1 & $-14,6$ & 1,20 & 1,10 & 1,33 & 1,37 & $\downarrow$ & $\uparrow$ \\
\hline 10 a 12 años & 4,5 & 4,1 & $-8,9$ & 0,90 & 1,10 & 1,25 & 1,37 & $\uparrow$ & $\uparrow$ \\
\hline $13 y+$ años & 3,6 & 3,0 & $-16,7$ & 0 & 0 & 1,00 & 1,00 & & \\
\hline \multicolumn{10}{|c|}{ Neonatal Tardía } \\
\hline Total & 1,3 & 1,1 & $-15,4$ & & & & & & \\
\hline 0 a 3 años & 2,6 & 1,4 & $-46,2$ & 1,80 & 0,70 & 3,25 & 2,00 & & \\
\hline 4 a 6 años & 2,1 & 1,7 & $-19,0$ & 1,30 & 1,00 & 2,63 & 2,43 & $\downarrow$ & $\downarrow$ \\
\hline 7 a 9 años & 1,5 & 1,5 & 0,0 & 0,70 & 0,80 & 1,88 & 2,14 & $\uparrow$ & $\uparrow$ \\
\hline 10 a 12 años & 1,2 & 1,1 & $-8,3$ & 0,40 & 0,40 & 1,50 & 1,57 & - & $\uparrow$ \\
\hline $13 y+$ años & 0,8 & 0,7 & $-12,5$ & 0 & 0 & 1,00 & 1,00 & & \\
\hline \multicolumn{10}{|c|}{ Post neonatal } \\
\hline Total & 4,1 & 3,1 & $-24,4$ & & & & & & \\
\hline 0 a 3 años & 7,9 & 8,1 & 2,5 & 6,30 & 6,80 & 4,9 & 6,2 & & 1 \\
\hline 4 a 6 años & 7,4 & 6,1 & $-17,6$ & 5,80 & 4,80 & 4,6 & 4,7 & $\downarrow$ & $\uparrow$ \\
\hline 7 a 9 años & 5,8 & 4,4 & $-24,1$ & 4,20 & 3,10 & 3,6 & 3,4 & $\downarrow$ & $\downarrow$ \\
\hline 10 a 12 años & 3,8 & 3,0 & $-21,1$ & 2,20 & 1,70 & 2,4 & 2,3 & $\downarrow$ & $\downarrow$ \\
\hline $13 y+$ años & 1,6 & 1,3 & $-18,8$ & 0 & 0 & 1,0 & 1,0 & & \\
\hline
\end{tabular}

Fuente: Elaboración propia a partir de los datos del MINSAL 2006. 
Tabla 4: Brechas absolutas y relativas de mortalidad infantil, según nueva agrupación de años de escolaridad materna, trienios 1998-2000 y 2001-2003

\begin{tabular}{|c|c|c|c|c|c|c|c|}
\hline $\begin{array}{l}\text { Grupos de } \\
\text { escolaridad } \\
\text { materna }\end{array}$ & $\begin{array}{l}\text { Tasa } \\
\text { de MI } \\
1990- \\
2000\end{array}$ & $\begin{array}{l}\text { Tasa } \\
\text { de MI } \\
2001- \\
2003\end{array}$ & $\begin{array}{c}\text { Cambio } \\
\%\end{array}$ & $\begin{array}{c}\text { RA } \\
\text { baja/alta } \\
1998- \\
2000\end{array}$ & $\begin{array}{c}\text { RA } \\
\text { baja/alta } \\
2001- \\
2003\end{array}$ & $\begin{array}{c}\text { RR } \\
\text { baja/alta } \\
1998- \\
2000\end{array}$ & $\begin{array}{c}\text { RR } \\
\text { baja/alta } \\
2001- \\
2003\end{array}$ \\
\hline Total & 9,9 & 8,2 & $-17,2$ & & & & \\
\hline 0 a 9 años & 13,4 & 11,4 & $-14,9$ & 7,40 & 6,40 & 2,23 & 2,28 \\
\hline 10 a 12 años & 9,5 & 8,2 & $-13,7$ & 3,50 & 3,20 & 1,58 & 1,64 \\
\hline $13 y+$ años & 6,0 & 5 & $-16,7$ & 0 & 0 & 1,00 & 1,00 \\
\hline
\end{tabular}

Fuente: Elaboración propia a partir del estudio MINSAL 2006.

po de escolaridad muy baja hasta los 9 años, se observa que el riesgo relativo en los dos períodos es prácticamente igual, mientras que el riesgo absoluto baja.

\section{Paso 5. Riesgo Atribuible Poblacional Porcentual (RAP\%)}

Según este indicador, en ambos períodos, alrededor de 39\% de las muertes infantiles se explicaría por la gradiente de escolaridad materna, es decir potencialmente se podría reducir la mortalidad infantil en esta proporción si todos los estratos educacionales tuvieran la tasa del grupo de menor riesgo, eliminando esta "exposición". En términos absolutos, significaría evitar 2.935 muertes infantiles de un total de 7.450 en el primer trienio y 2.309 muertes del total de 5.921 en el segundo trienio.

$$
\begin{aligned}
& \text { RAP\% } 1998-2000=((9,9-6) / 9,9) * 100=39,4 \% \\
& \text { RAP\% 2001-2003 }=((8,2-5) / 8,2) * 100=39 \%
\end{aligned}
$$

\section{Discusión}

El análisis realizado utiliza medidas epidemiológicas simples y ordena los pasos a seguir en el estudio básico y sencillo de desigualdades sociales en salud, desde el análisis de los números absolutos, la distribución de los grupos, los cálculos de los riesgos específicos y comparativos, la medición del efecto absoluto y relativo de las desigualdades $y$, finalmente, la estimación del impacto total de las desigualdades educacionales en la población infantil.
Del análisis de proporciones de nacimientos y muertes según grupo de escolaridad, especialmente la representación gráfica, se hace evidente la desproporcionalidad, observando una mayor proporción de muertes en relación a la distribución de los nacimientos para los tres grupos de menor escolaridad hasta los 9 años y una menor proporción para los dos grupos con mayores estudios. Además, destaca que el grupo de madres con 0-3 años es cada vez más pequeño y probablemente corresponde a mujeres que viven situaciones de vulnerabilidad social y marginalidad cada vez más extremas, por lo que la reducción de la mortalidad infantil en este grupo requiere un abordaje muy especial y focalizado. Probablemente debido a los resultados de las políticas sociales, este grupo tiene cada vez menos peso en la población y la reducción de la mortalidad infantil en este grupo impactará cada vez menos en la reducción global.

Del análisis de los cambios en las tasas de mortalidad infantil en estos dos períodos es importante destacar la clara reducción del riesgo infantil de morir para todos los grupos de escolaridad. Asimismo, en general disminuyen las tasas específicas por componentes de la mortalidad infantil, excepto para el grupo de 0 a 3 años de escolaridad materna en relación a la mortalidad neonatal precoz y post-neonatal. No obstante estas reducciones, persiste la gradiente social, en que el riesgo de morir es mayor en la medida que la escolaridad baja.

En el período analizado, el único componente de la mortalidad infantil donde el grupo de menor escolaridad materna disminuye los riesgos relativo y absoluto, es la mortalidad neonatal tardía (7 -27 días); hay que tener en cuenta, sin embargo, 
el escaso número de muertes neonatales tardías que hace inestable las estadísticas. Este componente está influido por el acceso y la calidad de la atención de salud y este resultado podría reflejar la mejoría en la atención perinatal, incluyendo la atención integral para los prematuros. Por otra parte, se observa un aumento importante del riesgo relativo de la mortalidad post-neonatal para este grupo (de 4,9 a 6,2), un componente que está asociado a las condiciones de vida. La mejoría de éstas corresponde a un trabajo de todos los sectores sociales y no sólo del sector salud.

Respecto a los otros estratos, se mantiene estable el riesgo relativo de mortalidad infantil para los grupos de escolaridad intermedio (7-9 años y 10-12 años). Al analizar los cambios por componente de los grupos intermedios, llama la atención que aumentan las brechas de mortalidad neonatal tardía pero se reducen para la postneonatal. Sería importante estudiar las causas de estas diferencias.

La redefinición del tramo de escolaridad muy bajo cambia el panorama sobre la situación de mortalidad infantil y desigualdades: en relación a la agrupación inicial, la tasa de mortalidad infantil específica es más baja, el riesgo relativo se mantiene y el riesgo absoluto disminuye. Estos cambios en los resultados resaltan la importancia que tiene la definición de los grupos. Para el análisis de desigualdades sociales, la definición de los grupos debe reflejar categorías de posición socioeconómica en que existe cierta homogeneidad respecto a las exposiciones y vulnerabilidades sociales.

Finalmente, los resultados de los pasos seguidos permiten matizar y comprender mejor los resultados de la evaluación de los Objetivos Sanitarios. Según estos, la brecha entre extremos educacionales habría aumentado; es decir, el efecto de la baja escolaridad en el diferencial de morir es mayor, porque este grupo es cada vez más marginal y el grupo de alta educación mejora cada vez más. Sin embargo, el peso que tiene el grupo de baja escolaridad en la población es cada vez menor, debido al efecto de otras políticas sociales, como la educación básica y secundaría universal y obligatoria, el sistema de protección social y el mayor acceso a la salud. Debido a este cambio en la distribución educacional de la población materna, la evolución del RAP\% que resume en un solo indicador el impacto global de las diferencias en el riesgo de morir de los estratos educacionales, muestra un impacto poblacional de la desigualdad social estable, con una muy leve mejoría.

\section{Referencias}

1. Minsal. Los Objetivos Sanitarios para la Década 2000 2010. Departamento de Epidemiología. Santiago, Chile: Ministerio de Salud; 2002. Disponible en: [Consultado el 27 de diciembre de 2009].

2. Lagos R. Mensaje de S.E. el Presidente de la República con el cual se envió al Congreso el proyecto de ley que establece un Régimen de Garantías en Salud. Presidencia; 22 de mayo de 2002. Disponible en http//.www.bcn. cl/histley/19966/index_html [Consultado el 28 de junio de 2008].

3. Whitehead M. The concepts and principles of equity and health. Discussion Paper. Programme on Health Policies and Planning. Copenhagen: WHO Regional Office for Europe; 1991. Disponible en: http://www.euro.who.int/ Document/PAE/conceptsrpd414.pdf [Consultado el 27 de diciembre de 2009].

4. MINSAL. Evaluación Objetivo III: Disminuir las desigualdades en salud. Objetivos Sanitarios para la Década 2000-2010. Evaluación a mitad del período. Departamento de Epidemiología. Santiago, Chile: Ministerio de Salud; 2006. Disponible en: www.epi.minsal.cl [Consultado el 30 de septiembre de 2009].

5. Hertel-Fernández AW, Giusti AE, Sotelo JM. The Chilean infant mortality decline: improvement for whom? Socioeconomic and geographic inequalities in infant mortality, 1990-2005. Bulletin of the World Health Organization. October 2007; 85: 798-804.

6. González R, Requejo JH, Nien JK, Merialdi M, Bustreo F. Tackling health inequities in Chile: maternal, newborn, infant, and child mortality between 1990 and 2004. Am J Public Health 2009; 99: 1220-6.

7. Mackenbach J, Kunst A. Measuring the magnitude of socio-economic inequalities in health: an overview of available measures illustrated with two examples from Europe. Social Science and Medicine 1997; 44: 6: 757-71. Disponible en: Social Science \& Medicine. [Consultado el 27 de septiembre de 2009].

8. Shaw M, Galobardes B, Lawlor DA, Lynch J, Wheeler B, Davey Smith G. The handbook of inequality and socioeconomic position. Bristol, UK: The Policy Press; 2007.

9. Schneider MC, Castillo-Salgado C, Bacallao J, Loyola E, Mujica O, Vidaurre M, et al. Métodos de medición de las desigualdades de salud. Rev Panam Salud Publica 2002; 12:6. Disponible en: http://www.scielosp.org [Consultado el 27 de septiembre de 2009]. 